\title{
Temporal Estimation of the 3d Guide-Wire Position Using 2d X-ray Images
}

\author{
Marcel Brückner ${ }^{1}$, Frank Deinzer ${ }^{2}$, and Joachim Denzler ${ }^{1}$ \\ 1 Chair of Computer Vision, Friedrich Schiller University of Jena, Germany \\ \{marcel.brueckner, joachim.denzler\}@uni-jena.de \\ 2 Siemens AG, Healthcare Sector, Germany and University of Applied Sciences \\ Würzburg-Schweinfurt, Germany \\ frank.deinzer@fhws.de
}

\begin{abstract}
We present a method for realtime online $3 \mathrm{~d}$ reconstruction of a guide-wire or catheter using $2 \mathrm{~d} \mathrm{X}$-ray images, which do not have to be recorded from different viewpoints. No special catheters or sensors are needed. Given a $3 \mathrm{~d}$ patient data set and the projection parameters, we use recursive probability density propagation to estimate a probability distribution of the current positions of guide-wire parts. Based on this distribution, we extract the optimal guide-wire position using regularization techniques. We describe the guide-wire position by a uniform cubic B-spline. Experiments on simulated and phantom data demonstrate the high accuracy and robustness of our approach.
\end{abstract}

\section{Introduction}

Most of the interventions of vessel diseases are performed by using catheters or guide-wires. One of the main difficulties is the navigation of these wires through the vessel system to the affected vessel. The common way of doing this is using a contrast agent and subtracted 2d X-ray images from a multi-axis angiography system (Fig. 2, left). Obviously, navigating a wire in a complex vessel system (e.g. brain, Fig. 2, middle left) using 2d X-ray images is not an easy task. Very good anatomic knowledge and a proper spatial sense are required, since the Xray images do not contain any depth information. A 3d reconstruction of the current wire position inside of a $3 \mathrm{~d}$ patient data set would be a great support for this difficult navigation task. Uncertainties during the navigation could be easily dissolved by rotating or zooming the $3 \mathrm{~d}$ reconstruction.

We present a method for realtime online $3 \mathrm{~d}$ reconstruction of a wire in a $3 \mathrm{~d}$ patient data set (3d rotational angiography, 3DRA) using subtracted $2 \mathrm{~d} \mathrm{X}$ ray images. The projection parameters are well known and the X-ray images do not have to be recorded from different viewpoints. This is very important since physicians do not want to change their current projection to get a $3 \mathrm{~d}$ reconstruction of the guide-wire. They expect the reconstruction to be an additional feature of the single position fluoroscopy. Furthermore, we do not need special catheters or sensors. Given the reconstructed 3d patient vessel system, we use 

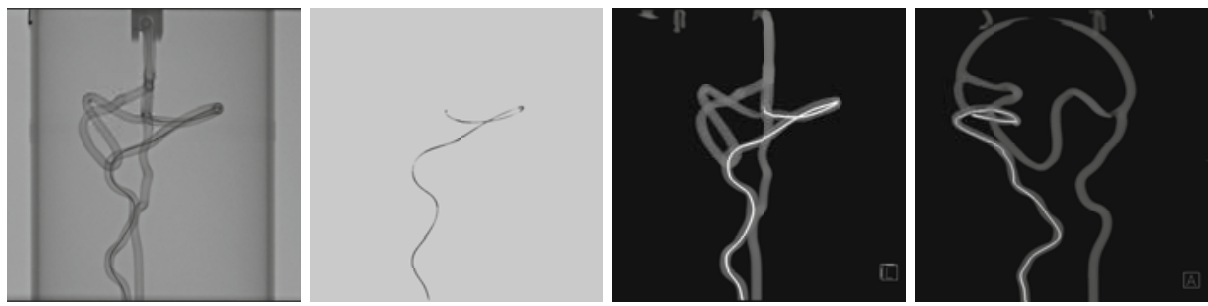

Fig. 1. Guide-wire in a vessel phantom (phantom1): native X-ray image (left), subtracted X-ray image (middle left) and two views of the $3 \mathrm{~d}$ reconstruction of the estimated wire in a 3DRA (middle right, right)

recursive probability density propagation [1] to build a $3 \mathrm{~d}$ probability distribution of the current $3 \mathrm{~d}$ position of wire parts. The representation and temporal update of this distribution is performed by using a particle filter [2]. We extract the optimal $3 \mathrm{~d}$ wire position using regularization techniques [3] based on this probability distribution. The wire is represented by uniform cubic B-splines 4. Fig. 11 shows an X-ray image (left), the subtracted image (middle left) and the resulting reconstruction of the estimated $3 \mathrm{~d}$ wire position inside of a 3DRA (middle right, right).

In the literature, one can find different approaches for the reconstruction of wires in a 3DRA. Solomon et al. [5] and Woods et al. [6] use special catheters and sensors to determine and visualize the $3 \mathrm{~d}$ position of a catheter tip. Bender et al. 7] use two or more X-ray images from different viewpoints to build a $3 \mathrm{~d}$ reconstruction of the guide-wire. By manually providing a starting point and direction of the wire, they reconstruct it iteratively using backprojection and comparing the backprojection to gradient images of the X-ray images. Baert et al. 8] use a calibrated biplane angiography system for $3 \mathrm{~d}$ reconstruction of the guide-wire by triangulation. Therefore they need an accurate tracking of the guide-wire in the 2d X-ray images. Point correspondences for triangulation are extracted by using the epipolar constraint. By using an angiography system and a 3DRA van Walsum et al. 9] estimate the 3d guide-wire position by reprojecting the segmented wire from the $2 \mathrm{~d}$ X-ray image. In opposite to our work, they do not use any temporal information for the $3 \mathrm{~d}$ position estimation. We also do not need any special hardware, manual interaction, or an accurate $2 \mathrm{~d}$ segmentation of the wire.

The remainder of this paper is structured as follows: In section 2 we describe our approach for estimating the $3 \mathrm{~d}$ position of a wire using $2 \mathrm{~d}$ X-ray images. We present different experiments on simulated and phantom data in section 3 . Conclusions are given in section 4 .

\section{Method}

The method we propose consists of two parts. First, we build with every new X-ray image a probability distribution of the currently possible $3 \mathrm{~d}$ positions of 
wire parts using the 3DRA, the prior knowledge of the wire position, i. e. the probability distribution of the last timestep, and the prior $2 \mathrm{~d} \mathrm{X}$-ray images. This is done by recursive probability density propagation using a particle filter [2]. In combination with a state estimator (e.g. maximum a posteriori or maximum likelihood), a particle filter is a powerful tool for state estimation. Platzer et al. 10. use a particle filter for $3 \mathrm{~d}$ blood flow reconstruction from $2 \mathrm{~d}$ angiograms. Unfortunately, using a particle filter for state estimation is - due to practical reasons - only possible if the state space is low dimensional. Since a generic, complex $3 \mathrm{~d}$ path of a wire cannot be described by just a few parameters, it is not promising to apply regular state estimation techniques. However, a particle filter still provides an efficient way to describe and update multi-modal probability distributions. The second part of our method consist of extracting the optimal wire position from this probability distribution using regularization techniques.

\subsection{Probabilistic Estimation of the 3d Wire Position}

Projecting $3 \mathrm{~d}$ data into a $2 \mathrm{~d}$ image plane causes information loss and creates ambiguities. Recursive probability density propagation [1] allows dissolving such ambiguities by integrating temporal information. In the context of our problem, it can be written as

$$
p\left(\boldsymbol{x}_{t} \mid\langle\boldsymbol{o}\rangle_{t}\right) \propto \underbrace{p\left(\boldsymbol{o}_{t} \mid \boldsymbol{x}_{t}\right)}_{\text {likelihood }} \int \underbrace{p\left(\boldsymbol{x}_{t} \mid \boldsymbol{x}_{t-1},\langle\boldsymbol{o}\rangle_{t-1}\right)}_{\text {update }} \underbrace{p\left(\boldsymbol{x}_{t-1} \mid\langle\boldsymbol{o}\rangle_{t-1}\right)}_{\text {recursion }} \mathrm{d} \boldsymbol{x}_{t-1} .
$$

Where $\boldsymbol{x}_{t} \in \mathbb{R}^{3}$ is some point in the $3 \mathrm{DRA}$ and $\langle\boldsymbol{o}\rangle_{t}=\left\langle\boldsymbol{o}_{1}, \boldsymbol{o}_{2}, \ldots, \boldsymbol{o}_{t}\right\rangle$ are the observations, i. e. subtracted X-ray images of the wire, until some point in time $t$. Recursive probability density propagation consists of three parts. The recursion $p\left(\boldsymbol{x}_{t-1} \mid\langle\boldsymbol{o}\rangle_{t-1}\right)$ is the reason why only the current observation is needed to update the distribution, i. e. the knowledge of the last timestep. Due to a lack of information we choose the initial $3 \mathrm{~d}$ wire distribution $p\left(\boldsymbol{x}_{0}\right)$ to be a uniform distribution inside of the patient vessel system.

In order to predict, where wire parts could move to in the next time step, the update probability distribution

$$
p\left(\boldsymbol{x}_{t} \mid \boldsymbol{x}_{t-1},\langle\boldsymbol{o}\rangle_{t-1}\right) \propto \frac{\mathcal{N}\left(d\left(\boldsymbol{x}_{t}, \boldsymbol{x}_{t-1}\right) \mid \mu, \sigma^{2}\right)}{\int_{\boldsymbol{\epsilon} \in \Omega} \int \mathcal{N}\left(d\left(\boldsymbol{x}_{t}, \boldsymbol{x}_{t-1}\right) \mid \mu, \sigma^{2}\right) p\left(\boldsymbol{x}_{t-1} \mid\langle\boldsymbol{o}\rangle_{t-1}\right) \mathrm{d} \boldsymbol{x}_{t-1} \mathrm{~d} \boldsymbol{\epsilon}},
$$

is used. In the literature [1, the update distribution does not depend on the old observations $\langle\boldsymbol{o}\rangle_{t-1}$. However, eq. (1) can be easily transformed to integrate the old observations into the update distribution. The normal distribution in the numerator and denominator with mean $\mu$ and variance $\sigma^{2}$ describes where wire parts could move to, given their current position. Important for this distribution is the shortest distance $d\left(\boldsymbol{x}_{t}, \boldsymbol{x}_{t-1}\right)$ between the past and the current point considering the vessel structure. This shortest distance calculation is based on the 3DRA using graph-theoretical methods. Since we suppose that the wire never 
leaves the vessel system, the distance to some point $\boldsymbol{x}_{t}$ outside of it is infinite. The denominator of eq. (2) prevents single high peaks in the $3 \mathrm{~d}$ wire probability. This is achieved by decreasing the probability that wire parts move to positions where the $3 \mathrm{~d}$ wire probability is already high. This normalization is restricted to a local space $\Omega$ around $\boldsymbol{x}_{t}$. The size of this space affects the smoothing. Note that this is very important, since otherwise single parts of the wire would get a much higher probability than other parts, which conflicts the prior information that each part of the wire should have the same probability. For the realisation using a particle filter, this means that it might happen that the particles tend to cluster at a small space instead of along the complete wire. This is because with every iteration more particles are drawn from the area with the higher probability.

The third part of the recursive probability density propagation is the likelihood

$$
p\left(\boldsymbol{o}_{t} \mid \boldsymbol{x}_{t}\right) \propto \max _{\left(i^{*}, j^{*}\right)} \boldsymbol{o}_{t}\left(i^{*}, j^{*}\right) \mathcal{N}\left(i-i^{*}, j-j^{*} \mid \mathbf{0}, \boldsymbol{\Sigma}\right)
$$

which is used to integrate the actual observation $\boldsymbol{o}_{t}$, i. e. the subtracted X-ray image, into the $3 \mathrm{~d}$ wire probability distribution. Where $(i, j)$ are the image coordinates of the projected $3 \mathrm{~d}$ point $\boldsymbol{x}_{t}$ and $\boldsymbol{o}_{t}(i, j)$ is the gray value of the observation at this position weighted by a Gaussian kernel $\mathcal{N}(\cdot, \cdot \mid \mathbf{0}, \boldsymbol{\Sigma})$. The projection parameters are known by the angiography system which recorded the $2 \mathrm{~d}$ X-ray image. In order to prevent higher probabilities in areas where the projected wire is crossing itself, we use the maximum instead of a sum.

\subsection{Extraction of the Optimal Wire}

We use uniform cubic 3d B-splines [4] $\mathbf{S}_{\mathcal{C}}(r):[0,1] \rightarrow \mathbb{R}^{3}$ to describe the $3 \mathrm{~d}$ wire position. The appearance of a B-spline is defined by the position of its control points $\mathcal{C}=\left\{\boldsymbol{c}_{0}, \boldsymbol{c}_{1}, \ldots, \boldsymbol{c}_{m}\right\}, \boldsymbol{c}_{k} \in \mathbb{R}^{3}$. As start and end of the spline, we use the first and last control point, respectively: $\mathbf{S}_{\mathcal{C}}(0)=\boldsymbol{c}_{0}, \mathbf{S}_{\mathcal{C}}(1)=\boldsymbol{c}_{m}$.

Given the probability distribution $p\left(\boldsymbol{x}_{t} \mid\langle\boldsymbol{o}\rangle_{t}\right)$, which is caused by the wire, we want to extract the $3 \mathrm{~d}$ wire position, i. e. the spline control points. Obviously this is an ill-conditioned problem which cannot be solved without specifying further constraints. But it can be solved using regularization techniques [3]. We search for the optimal spline control points

$$
\hat{\mathcal{C}}=\underset{\mathcal{C}}{\operatorname{argmax}} \underbrace{\int_{0}^{1} \log p\left(\mathbf{S}_{\mathcal{C}}(r) \mid\left\langle\mathbf{o}_{t}\right\rangle\right) \mathrm{d} r}_{\text {probability }}+\alpha \underbrace{\int_{0}^{1}\left|\mathbf{S}_{\mathcal{C}}^{\prime}(r)\right| \mathrm{d} r}_{\text {length }}-\beta \underbrace{\int_{0}^{1} \mathbf{S}_{\mathcal{C}}^{\prime \prime}(r)^{2} \mathrm{~d} r}_{\text {smoothness }}
$$

by maximizing an objective function which consists of three parts. The searched spline should stride an area with high probability and maximize length and smoothness. The parameters $\alpha$ and $\beta$ weight the different parts of the objective function. Since the probability of any $3 d$ point outside of the vessel system is zero, the spline cannot leave it. We use Powell's method [1] for optimization. 


\section{Experiments}

We tested our method on two simulated sequences based on the 3DRA of a half brain (sim1 and sim2, 300 images each) and on two sequences using real X-ray images (Fig. 1. left) of a vessel phantom (phantom1, 186 images and phantom2, 231 images). Fig. 2 (middle left, middle right) shows the two 3d data sets. Each of these data sets is scaled to a size of $256^{3}$ voxels resulting in a voxel size of $0.765 \mathrm{~mm}$ for the phantom and $0.539 \mathrm{~mm}$ for the brain data set. An impression of the different sequences is given in Fig. 11and Fig. 2 (right). Each of these images shows the estimated $3 \mathrm{~d}$ wire position after the last image from the viewpoint where the $2 \mathrm{~d}$ X-ray images of the respective sequence are taken. The $3 \mathrm{~d}$ data sets and the X-ray images are recorded on a Siemens Artis zee C-arm system (Fig. 2 (left), image resolution: $1240 \times 960$, pixel size $0.308 \mathrm{~mm} \times 0.308 \mathrm{~mm}$ ). We use a $33 \times 33$ Gaussian kernel to build the 2 d probability distribution of eq. (3). We choose $\mu=7.5$ and $\sigma^{2}=2.5$ for eq. (2) and our particle set consists of 15000 particles. To reach realtime performance in our experiments, we use a graph, which is based on the thinned vessel system of the 3DRA, for the distance calculation inside of the vessel system. This graph is also used to find the longest possible wire given the $3 \mathrm{~d}$ wire probability distribution what simplifies the extraction of the optimal wire and to decide how many spline control points are necessary for the wire representation.

In order to show the accuracy of our method, we analyze the error of the wire tip and the complete wire. The ground truth $3 \mathrm{~d}$ and reprojected $2 \mathrm{~d}$ wire position of the simulated sequences are well known. For the phantom sequences only the manually extrated $2 \mathrm{~d}$ ground truth is given. Thus we analyze just the accuracy of the reprojected wire of these sequences. We calculate the $2 \mathrm{~d}$ and $3 \mathrm{~d}$ Euclidean distance between the estimated wire tip and the ground truth wire tip. To evaluate the error of the complete wire, we calculate the shortest Euclidean distance between each pixel (voxel) of the estimated wire to some pixel (voxel) of the ground truth wire. Note that this evaluation rates an estimated wire which is too short in comparison to the ground truth wire too good. However, this case
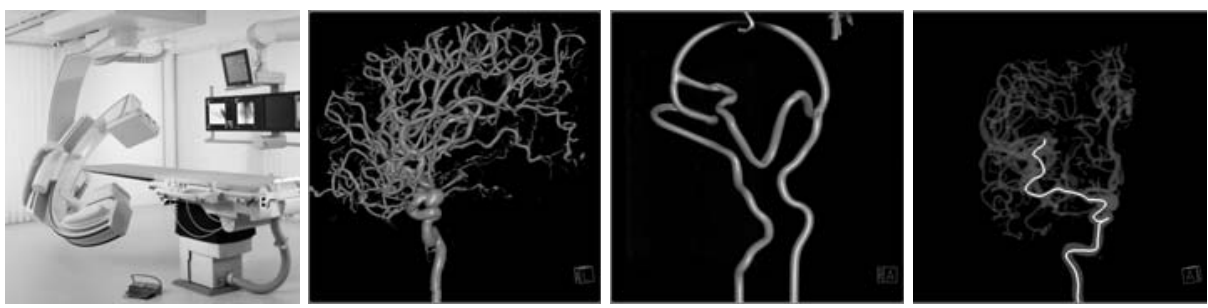

Fig. 2. Siemens Artis zee C-arm system (left, www.siemens.com). 3d half brain data set used for the simulated (middle left) and the $3 \mathrm{~d}$ phantom data set used for the phantom sequences (middle right). $3 \mathrm{~d}$ visualization of the estimated wire after the last image of the sequence sim1 of subtracted X-ray images (right). The viewpoint of this image is similar to the viewpoint where the X-ray images are taken. 

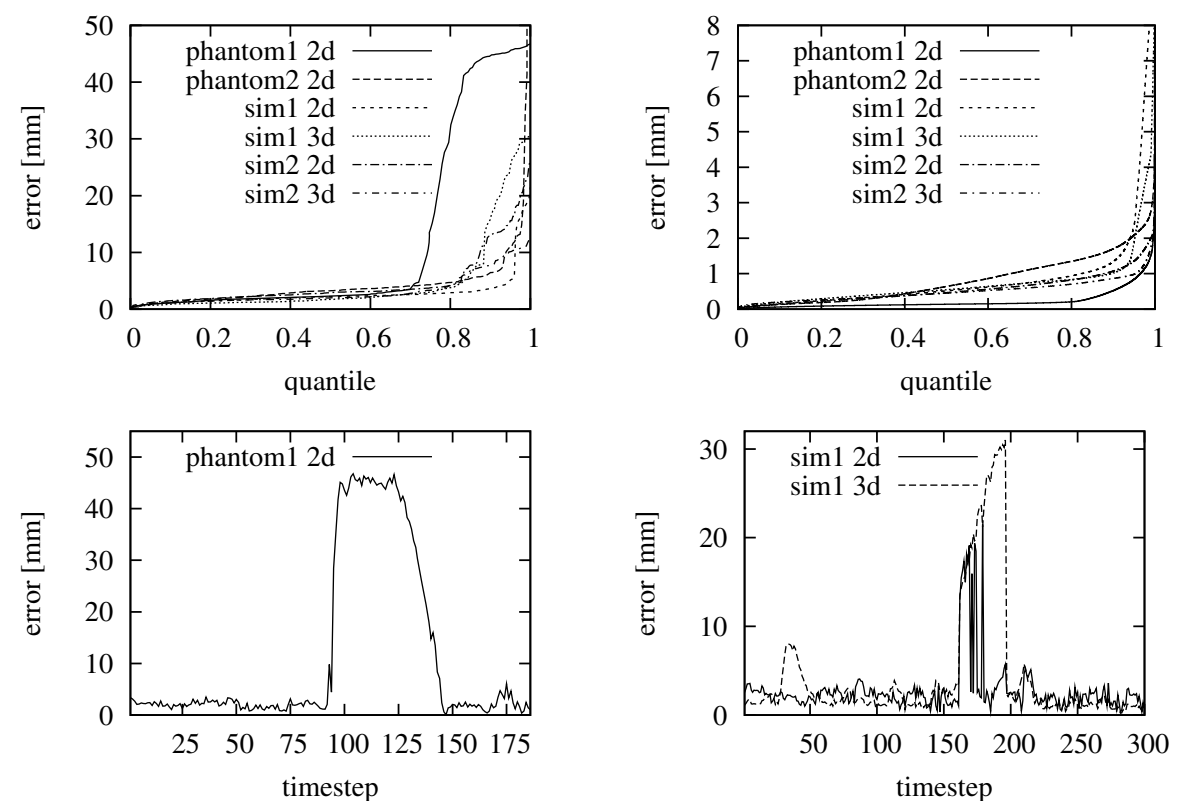

Fig. 3. Quantile plots of the $2 d$ and $3 d$ (only simulated sequences) error of the wire tip (top left) and the complete wire (top right) of each sequence. Error of the wire tip for each timestep of the sequences phantom1 (bottom left) and sim1 (bottom right). The overall median (quantile 0.5) error for the wire tip is $2.52 \mathrm{~mm}$ and $0.47 \mathrm{~mm}$ for the complete wire. Explanations for temporarily larger errors are given in section 3.1

is handled by the wire tip error. Since the metric sizes of each pixel and voxel are known, we present the distances in millimeters.

Fig. 3 shows quantile plots of the wire tip error (top left) and of the complete wire error of each sequence (top right). An overview of the median (quantile 0.5) errors of each single sequence and of all sequences (all) is presented in Table 1 The median error of the reprojected wire tip is $2.52 \mathrm{~mm}$ and $0.47 \mathrm{~mm}$ for the complete wire. In section 3.1, we describe reasons for temporarily larger errors. Our approach takes 1.48 seconds for processing a timestep on an Intel Pentium $42.8 \mathrm{GHz}$ with $1 \mathrm{~GB}$ RAM. Note that since a particle filter can be completely parallelized, much better runtimes are possible on a modern multi core system using an optimized implementation.

\subsection{Reasons for Inaccuracies}

A reason for small errors are ghosting artefacts. These artefacts are created by the X-ray detector due to a temporal averaging of the X-ray images. An example for a ghosting artefact is displayed in Fig. 4 (left). Because the wire is moved quite fast it seems to have two tips. Also most of the time the estimated wire tip position is slightly in front of the real wire tip position, since eq. (4) benefits long wires and the observation is slightly blurred. 
Table 1. Median errors in millimeters for each single and for all sequences (all)

\begin{tabular}{lccccc}
\hline error $[\mathrm{mm}]$ & phantom1 & phantom2 & sim1 & sim2 & all \\
\hline cath. tip 2d (3d) & 2.27 & 3.24 & $2.22(1.71)$ & $2.90(1.98)$ & $2.52(1.88)$ \\
catheter 2d (3d) & 0.14 & 0.64 & $0.52(0.55)$ & $0.47(0.45)$ & $0.47(0.50)$ \\
\hline
\end{tabular}
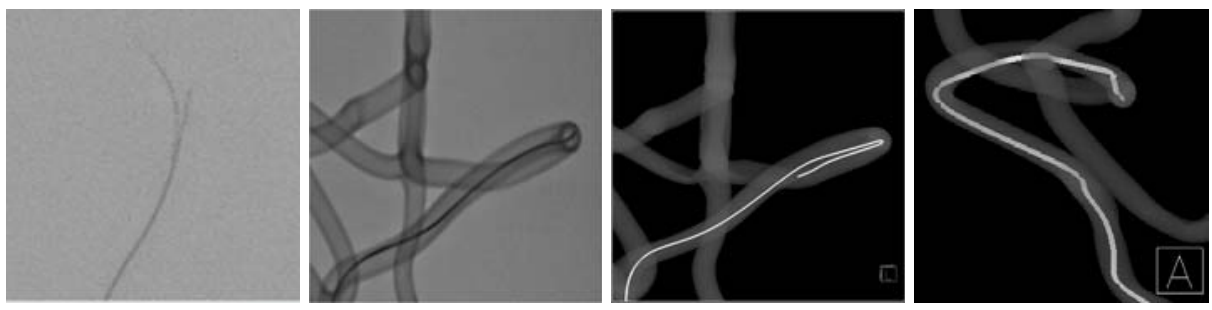

Fig. 4. Ghosting artefact (left), example for an ambiguity in phantom1: X-ray image (middle left), (incorrectly) estimated wire (middle right, right)

Most of the larger errors can be explained by ambiguities of the $3 \mathrm{~d}$ catheter position in the $2 \mathrm{~d}$ X-ray images. Fig. 3 (bottom left) shows the $2 \mathrm{~d}$ error of the wire tip for each timestep of the phantom1 sequence. Most of the time, this error is really low, but in the second part of the sequence, the error gets suddenly very large. The reason for this is the ambiguity shown in Fig. 4 (middle left). Because of an overlapping vessel, the $3 \mathrm{~d}$ position of the wire cannot be estimated correctly. The (incorrectly) estimated wire is shown in Fig. 4 (middle right, right). Fig. 3 (bottom right) shows the $2 \mathrm{~d}$ and $3 \mathrm{~d}$ error of the wire tip in sequence sim 1 . At the start and in the middle of this sequence, the $3 \mathrm{~d}$ error suddenly increases, while the $2 \mathrm{~d}$ error stays low. The reason for this is that the wire is moving in the direction of the optical axis of the X-ray detector. Hence the $3 \mathrm{~d}$ position of the wire tip cannot be estimated correctly. However, the reprojected $2 \mathrm{~d}$ position is still quite accurate.

Note that in each of these cases our method is robust enough to return very quickly to a high accuracy as soon as the ambiguities are resolved. Furthermore, many of these ambiguities can easily be resolved by using a second pair of X-ray source and detector with a different viewpoint, which could be easily provided by a biplane system. In this case, the likelihood in eq. (1) would consist of two observations.

\section{Conclusions}

We presented a new approach for realtime online 3d guide-wire reconstruction using $2 \mathrm{~d}$ X-ray images, which do not have to be recorded from different viewpoints. This meets the clinical needs since physicians do not want to change their current projection to get a $3 \mathrm{~d}$ reconstruction of the guide-wire. By using recursive probability density propagation, we built a $3 \mathrm{~d}$ probability distribution of the 
positions of wire parts based on a $3 \mathrm{~d}$ patient data set and $2 \mathrm{~d}$ X-ray images. In order to represent and update this multi-modal distribution, we used a particle filter. To extract the optimal $3 \mathrm{~d}$ wire position from this distribution, we applied regularization techniques. We described the $3 \mathrm{~d}$ wire position by a uniform cubic B-spline. Our method does not need any special catheters or sensors. Performing different experiments on simulated and phantom data showed the high accuracy of our method (Table 1). We achieved a median error of the reprojected wire tip of $2.52 \mathrm{~mm}$. The median error of the reprojected complete wire was $0.47 \mathrm{~mm}$. The achieved median $3 \mathrm{~d}$ error is $1.88 \mathrm{~mm}$ for the tip and $0.50 \mathrm{~mm}$ for the complete wire.

In our future work, we will improve the accuracy of our approach by using a biplane angiography system. In order to better model the guide-wire behaviour we will also add a speed estimation of the guide-wire tipp. We will also implement our approach using multi processing techniques. The clinical evaluation of our approach is imminent.

\section{References}

1. Doucet, A., DeFreitas, N., Gordon, N.: Sequential Monte Carlo Methods in Practice. Springer, Heidelberg (2001)

2. Arulampalam, M., Maskell, S., Gordon, N., Clapp, T., Sci, D., Organ, T., Adelaide, S.: A tutorial on particle filters for online nonlinear/non-Gaussian Bayesian tracking. IEEE Trans. on Signal Processing 50(2), 174-188 (2002)

3. Engl, H., Hanke, M., Neubauer, A.: Regularization of Inverse Problems. Kluwer Academic Publishers, Dordrecht (1996)

4. de Boor, C.: A Practical Guide to Splines. Springer, Heidelberg (1978)

5. Solomon, S., Dickfeld, T., Calkins, H.: Real-Time Cardiac Catheter Navigation on Three-Dimensional CT Images. J. of Interv. Card. Electrophys. 8(1), 27-36 (2003)

6. Wood, B., Zhang, H., Durrani, A., Glossop, N., Ranjan, S., Lindisch, D., Levy, E., Banovac, F., Borgert, J., Krueger, S., et al.: Navigation with Electromagnetic Tracking for Interventional Radiology Procedures: A Feasibility Study. J. of Vascular and Interv. Radiology 16(4), 493-505 (2005)

7. Bender, H.J., Männer, R., Poliwoda, C., Roth, S., Walz, M.: Reconstruction of 3D Catheter Paths from 2D X-ray Projections. In: Proc. of the Second Int. Conference on Med. Image Computing and Computer-Assisted Intervention, pp. 981-989 (1999)

8. Baert, S., van de Kraats, E., van Walsum, T., Viergever, M., Niessen, W.: Threedimensional guide-wire reconstruction from biplane image sequences for integrated display in 3-D vasculature. IEEE Trans. on Med. Imaging 22(10), 1252-1258 (2003)

9. van Walsum, T., Baert, S., Niessen, W.: Guide wire reconstruction and visualization in 3DRA using monoplane fluoroscopic imaging. IEEE Trans. on Med. Imaging 24(5), 612-623 (2005)

10. Platzer, E.S., Deinzer, F., Paulus, D., Denzler, J.: 3D Blood Flow Reconstruction from 2D Angiograms. In: Bildverarbeitung für die Medizin 2008 - Algorithmen, Systeme, Anwendungen. Informatik aktuell, pp. 288-292. Springer, Heidelberg (2008)

11. Powell, M.J.D.: An efficient method for finding the minimum of a function of several variables without calculating derivatives. The Comp. J. 7(2), 155-162 (1964) 